$\angle 8$ Research Square

\title{
Spatial Ability in children with Attention-Deficit/Hyperactivity Disorder (ADHD) and its Impact on Executive Functions.
}

\author{
Solmaz Soluki \\ Shahid Beheshti University

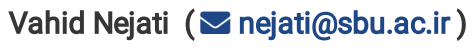 \\ Shahid Beheshti University https://orcid.org/0000-0003-0419-5207 \\ Jalil Fathabadi \\ Shahid Beheshti University
}

\section{Research article}

Keywords: Spatial ability, attention deficit hyperactivity disorder, executive functions, school- age children

Posted Date: July 15th, 2020

DOI: https://doi.org/10.21203/rs.3.rs-41923/v1

License: (c) (i) This work is licensed under a Creative Commons Attribution 4.0 International License. Read Full License 


\section{Abstract \\ Background}

Attention-Deficit/Hyperactivity Disorder (ADHD) is associated with deficits in cognitive functions. Spatial ability (SA) which plays a crucial role in different domains of academic and daily life is constructed with eight separate and unified factors.

\section{Method:}

The first objective of this study was to explore differences in SA factors in 128 school children of third to sixth grade with and without ADHD. The second objective of the study was to explore the SA factors' ability to predict the performance of ADHD participants in three executive functions. The Flexibility of Closure, Closure Speed, Perceptual Speed, Visualization, Spatial Relation, Spatial Orientation, Spatial Temporal, and Wayfinding were measured through eight tasks.

\section{Results}

Participants with ADHD were less accurate in all the tasks except Spatial Temporal task, and their reaction time was more in Visualization, Spatial Relation, and Spatial Orientation tasks comparing to children with typical development. Regression analysis exhibited that some of SA factors were able to predict working memory and cognitive flexibility, but they could not predict response inhibition.

\section{Conclusion}

Regarding these results, it seems necessary for specialists to include assessment of all SA factors and to train them in cognitive rehabilitation programs for children with ADHD.

\section{Introduction}

Attention-deficit/hyperactivity disorder (ADHD), a common neurodevelopmental disorder which is diagnosed by symptoms of inattention, hyperactivity, and impulsivity, can cause a deficit of activity in two or more settings of daily life in about 5 to $7 \%$ of children $(1,2)$.

At the cognitive level, symptoms of ADHD are often explained by dysexecutive functioning (3). Cognitive deficits such as deficits in attention and executive functions (EFs) have frequently been proved in ADHD people (3). Spatial ability (SA), one of the main cognitive functions, is probably defective in children with ADHD (4). A poor spatial skill can influence various aspects of a person's daily life, from problems with the ability to interpret graphs and tables, academic skills such as reading and mathematics to failures like getting lost or spending time to remember where things are (5-7).

The term of spatial ability (SA) is an umbrella term that refers to "the skill in representing, transforming, generating, and recalling symbolic, nonlinguistic information" (8). A theoretical framework for SA was presented by Uttal and colleagues (9). They stated that the spatial tasks could be divided along two dimensions, intrinsic-extrinsic dimension, and static-dynamic dimension, the first one differentiates spatial relations inherent to objects, such as the location of object parts, with spatial relations among objects and between objects and the larger space around them, such as wayfinding. While the second contrasts tasks based on whether they involve movement or transformation. Based on these two dimensions, a $2 \star 2$ classification of spatial skills was developed (intrinsic-static, intrinsic-dynamic, extrinsic-static, and extrinsic-dynamic, 9). Yilmaz collected eight factors from previous meta-analysis and factor-analysis, decided that these factors cover the whole nature and construction of SA (10). We assumed that based on the nature and construction of eight factors, three factors of Closure Speed (CS), Flexibility of Closure (FC), and Perceptual Speed (PS) can be located in the intrinsic-static cell, two factors of Visualization (VIS), and Spatial Relation (SR) in the intrinsic-dynamic cell, Spatial Orientation (SO) factor in the extrinsic-static, and Spatial Temporal ability (ST), and Wayfinding (WF) in the extrinsic-dynamic cell. (The definition of eight factors are mentioned in Table 1).

\section{Due to technical limitations, Table 1 is provided in the Supplementary Files section.}

It has been proven that the parietal lobe underlies different aspects of attention $(11,12)$, while its essential role in processing spatial information should be taken into account. It has been shown that different parts of the parietal lobe especially the posterior parietal cortex underpinned spatial ability tasks $(13,14)$. It is also notable that in the final level of perceptual processing, all of spatial information from other areas of brain come together in the inferior parietal lobule (IPL) to integrate the action and semantic knowledge required for complex tools used in daily life (15). At the neural level, structural brain imaging techniques were approved abnormalities in the parietal lobe in individuals with ADHD such as abnormal and smaller gray matter volumes $(16,17)$. Functional neuroimaging indicated that children with ADHD have less activation in the right parietal-occipital areas and the IPL while performing a mental rotation task (18), they also have hypoactivation in the left superior parietal lobe (SPL) while doing 
Raven's Standard Progressive Matrices (19), and dysfunction in posterior parietal regions during executing the Rapid Visual Information Processing, and Pattern Recognition Memory tasks from the CANTAB (20). Accordingly, it could be assumed that children with ADHD had difficulty at least in some of the SA factors.

A neuroimaging study found children with ADHD, compared with typically developing children, have weaker functional connectivity between prefrontal and parietal cortices (19). One of the prefrontal cortex functions is related to EFs. Three core EFs include working memory, cognitive flexibility, and response inhibition (3). It has been proposed that symptoms of ADHD stand up a central deficit in a specific EFs domain such as response inhibition or working memory (21-24). However, the performance relations and impacts of posterior parts of the brain and prefrontal cortex have not examined via behavioral experiments in children with ADHD.

Several behavioral studies have focused on SA impairments in children with ADHD (25-37), although some of the studies did not show the existence of any differences in spatial tasks between the performance quality of children with ADHD and typically developing children (38-42). The reason for many inconsistencies might be due to the fact that not only the unity of SA is not precisely defined, but also not even a single appropriate test is used to measure these factors in various researches. In respect to SA factors, few studies have focused on SA factors separately in ADHD children (30, 37 and, 39). It must be noted that VIS, SO, ST, and WF factors have not been investigated in this group of children yet. Therefore, examination of all SA factors using suitable tasks to measure the nature of each factor specifically, could increase our knowledge about the cognitive profile in children with ADHD. Furthermore, since there is a strong evidence of deficits in structure and activation of the parietal lob in children with ADHD, we hypothesized that SA was also defective in this group of children which can affect functions of the prefrontal cortex as well. Altogether, in this study we aimed to compare eight factors of SA between ADHD and typical developmental (TD) children. The second aim of the study was to examine the predictability of SA on three core EFs in ADHD TD and children

\section{Method \\ Participants}

One hundred twenty-eight children aged between 9 to 12 years old participated in this cross-sectional study as two TD and ADHD groups. They were selected in Tehran city during the autumn and winter of 2019. Sixty four children (14 females and 50 males) with the mean age of 10.02 (0.98), were diagnosed to be ADHD (combined type) by two psychiatrists according to the Diagnostic and Statistical Manual of Mental Disorders Fifth Edition (1) criteria were put in ADHD group. They were selected through purposeful sampling among children who their parents filled an informed consent form, Demographic information questionnaire, and Conners' Parent Rating Scale (short form; 43). TD group included 64 children (29 females and 35 males) with the mean age of 9.97 (0.99). Multi stage sampling was done for selecting TD children from two elementary schools. A letter was sent to more than 10 schools to recruit the required number of participants. After the initial contact, the two schools which agreed to participate in the study selected randomly. They were received a formal letter, an informed consent form, Demographic information questionnaire, and Conners' Parent Rating Scale (43) and sent them for parents. Finally, researchers selected the samples randomly among children who their parents were volunteer and filled all of the forms. The inclusion criteria for the ADHD group was getting scores above 60 in short form version of the Conners' Parent Rating Scale (43) and for TD group was getting scores below 38 on this scale (44). The exclusion criteria for both groups included intellectual disability, autism spectrum disorder, physical conditions (i.e., visual, hearing, heart, rheumatic, orthopedic), neurological disorder, and regular use of medication except Methylphenidate. Psychiatrists decided about exclude children from ADHD group. For TD group, researchers investigated information available at schools (children's records) in order to insure they did not suffer from significant psychological or physical deficits.

Although for regression analysis we needed 80 children in each groups (power $=0.80$ ) but we were not able to replace the excluded samples because of our time limitation.

\section{Material And Method}

\section{Conner's Parent Rating Scale-48 (43)}

This is a 48-items questionnaire, which includes 6 hyperactivity factors including attention deficit, behavioral disorder, impulse disorder, hyperactivity, psychosomatics disorder, and anxiety disorders. In a study by Khoushabi, validity of the questionnaire was estimated by Cronbach alpha equal to $93 \%$ in the Iranian population (44).

\section{Battery of SA task (45)}

This battery comprises eight modified computerized tasks which assess all the factors of SA separately (See Table 1). The Accuracy score (ACC), and Reaction Time of correct responses (RT) were calculated in this battery as variables. In a recent study, the Test-retest reliability of ACC (0.689 to 0.992), and RT (0.963 to 0.997$)$ were reported high and very high for all tasks (45). The tasks are described as follows: 
Flexibility of Closure task. This task is a modified version of the figure-ground discrimination subtest of the Test of Visual Perception Skills-Revised (TVPS-R; 46). It includes 16 items with two practice items. During a test, a shape is displayed on the top of the screen and four patterns with complicated and conglomerated ground present on the bottom. Participants are asked to found shape in one of the four patterns and press the number of correct answer on keyboard. Response time for selecting an answer to each item is restricted to 10 seconds.

Closure Speed task. This task is a modified version of the visual closure subtest of the TVPS-R (46). It consists of 16 items with two practice items. An incomplete shape is presented on the top of the screen and four complete shapes are placed on the bottom of screen for each item. Participants should imagine the completed shape and match it with one of the shapes on the bottom of screen and press the number of correct answer on keyboard. Response time to each item is restricted to 10 seconds.

Perceptual Speed task. This task is a modified version of the Visual discrimination subtest of TVPS-R (46). It comprises 16 items with two practice items. A shape is displayed on the top of the screen and the participant should determine which of five shapes on the bottom are exactly the same as the shape on the top of the screen and press the number of correct answer on keyboard. Response Time to each item is restricted to 10 seconds.

Visualization task. This task is a modified version of the paper folding task (47). The task consists of 16 items with two practice items. During this task, participants should imagine folds applied to a piece of paper, without any representation of the folding action itself. The paper used is colored differently on each side to make the back and forth sides distinct. Each of the 16 items represented a different paper shape. Orientation of folding on paper is specified by an arrow and location of folding is marked by dotted lines. Participants are asked to visualize that the folded paper accordingly and select the matching picture among the four pictures. The choices remain on screen until the participants' response and the response time is recorded by the program.

Spatial Relation task. This task is a modified version of the Animal mental rotation task (48). It consists of 48 items with two practice trials. Participants are asked to find out if the two shown animal stimuli are identical or mirror images of each other. The stimuli include figures of three different animals (elephant, leopard, and horse). On a given trial, an animal is presented twice: A standard figure of an upright-standing animal is shown on the left side while a comparison figure is on the right side. In half of the trials, the comparison figure is rotated in picture plane and identical (which are called as "same trials") and in the other half of trials, the comparison figure is mirror-reversed (which are called as "different trials"). Half of the standard figures are presented facing left, the other half facing right. The angular disparity between the two figures (presented on the left and right side of the screen) is $22.5^{\circ}, 67.5^{\circ}, 112.5^{\circ}$, or $157.5^{\circ}$ clockwise and counter-clockwise (i.e., $202.5^{\circ}, 247.5^{\circ}, 292.5^{\circ}$, or $337.5^{\circ}$ ). In each of the eight angular disparities, each pair of drawings is presented twice (once in a 'same' and once in a 'different' trial). At the beginning of each trial, a 5-centimeters grey fixation square is presented for 500 milliseconds followed by the two stimuli figures which want the participant to answer the item by pressing key number 1 for "same" and key number 2 for "different". Response time to each trial is restricted to 10 seconds.

Spatial Orientation task. Picture task was applied for measuring SO factor. This task developed by Zoest (49) based on Hegarty and Waller (50) work. In each item of this task a picture is shown with one photographer standing in a scene with a lot of objects. The photographer in the picture is taking a photo of what he can see from his perspective. Next to the picture, three photos are shown. For each photo, the participant has to detect the matched picture based on the perspective of the photographer. Item remains until responses and the ACC and RT are recorded by the program.

Spatial Temporal task. This task is a modified version of the interception task (51). This task is designed to assess the participants' ability to combine the speed and path extrapolation. The task includes a game-like interface in which a small circular target moves horizontally across the top of the screen at one of the three preset speeds (175, 200, and 250 pixel/second). In this task, the participants should try and 'hit' the moving circular target using a 'missile' that is launched from the lower right corner of the screen by pressing the spacebar on the keyboard. When the spacebar is pressed, the missile moves straight up at a constant velocity equals to $175 \mathrm{px} / \mathrm{sec}$ to the point of intersection that is exactly 350 pixels away from the launch point. Given the constant velocity of the missile, it takes 2 seconds for the missile to reach the intersection point. This is the same across all trials. To successfully hit the target, the participants must release the missile (by pressing the spacebar) on time so that any point of the circle (e.g., target) crosses the point of intersection. To make the task more difficult, an extra waiting time of 575 milliseconds was added to each of three preset speeds, therefore the overall starting waiting time (and hence, the initial starting point) for each circular target is increased, resulting in 3 different trial types overall. Participants are asked to complete each of these 3 trials for 7 times, resulting in 21 trials overall. Before beginning the actual trials Participants are given 5 random practice trials with varying speeds and waiting times to get themselves familiar with the whole interface.

Wayfinding task. This task was adapted from Mengue-Topio, Courbois, Farran, and Sockeel (52). It has three phases, including Memory for Landmarks (ML), Learning the Path (LP), and Shortcut Distance (SD). The experimental virtual environment, required in this task is comprised of a 4 * 4 regular grid of streets lined with high brick walls. This virtual space is surrounded by distant landscapes providing no distinctive cues which could be of help for the participants in wayfinding. Three buildings and 17 landmarks are located in different places of the space. The buildings are a "railway station" (A), a "store" (B), and an "apartment building" (C). This is projected onto a 1.20 * 1.50 meter screen. The distance between the screen and the participants is 2 meters. Participants can explore the virtual town using a keyboard and a mouse. Pressing the backspace key on keyboard affected forward movement and moving the mouse to the right or left side, controlled rotational movements inside the space. Participants start navigation of the virtual environment from a first-person viewpoint at a constant velocity. The sum of landmarks correctly

Page $4 / 15$ 
recognized by the participants in both paths is considered as a score of memory for the landmark phase (ACC). The Number of Trials (NT) performed to reach the criteria on paths is added up for scoring of the learning phase. In the shortcut phase, the walked distance between two buildings (meters) is calculated to identify the shortest path, therefore there are three variables for analysis in this task.

\section{N-Back task}

This task has been used in the assessment of working memory performance $(53,54)$. In the 1 - back task, used in the present study, participants are shown a sequence of stimuli and they have to indicate if the current stimulus matches the previous one. This Performance is assessed in terms of accuracy. The validity of the task is reported between 0.29 to 0.91 in different studies (55).

\section{Wisconsin Card Sorting Test (WCST)}

This test was first designed by Grant and Berg and used for the measurement of cognitive flexibility (56). The participants are asked to match several test cards to reference cards based on color, shape, or number of stimuli on the cards. After each matching, the provided feedback enables the participants to find or confirm the correct rule of classification. After a fixed number of correct matches, the rule changes without notice, and consequently, the participants must shift to a new model of classification. The number of categories correctly sorted based on correct responses and responses to a previously correct category can be classified as perseverative responses. In the present study, a computerized short form of test with 64 cards was used (57). The reliability and validity of the test have been proved (58).

\section{Go/No-Go task}

This task has been used for the measurement of response inhibition $(59,60)$. In this task, after a small plus in the middle of the computer screen as a fixation point, a plane appears for $500 \mathrm{~ms}$ and participants have to indicate the direction of the plane with one of four arrow keys on the keyboard during $2000 \mathrm{~ms}$. In some trials called No-Go, 20\% in the present study, one beep sound presented after the airplane and participants have to withhold the response in this trial. The measures of this task include reaction time and accuracy of Go trials and accuracy of No-Go trials.

\section{Procedure}

Parents have filled in the consent form, and Conner's scale after reading explanations about the objectives and procedure of the research. ADHD and TD children were tested individually in a quiet room. The Unity software was used to program and run the experiments. Each task was preceded by a practice phase, and before starting the test phase all children were asked to repeat the task instructions to ensure that they understood. All tasks were presented in the same order for all participants on a 15-inch PC screen. Children viewed from a distance of approximately $60 \mathrm{~cm}$. The battery of spatial ability tasks and executive function tasks administered to the children in three sessions of 40 minutes.

\section{Statistical Methods}

Statistical analysis was performed with SPSS version 22 (SPSS Inc., Chicago, IL, USA). Data were summarized using mean \pm standard deviations and $95 \%$ confidence intervals for continuous variables and percentages for categorical data. To assess the equality of two groups for gender ratio, Chi-Square Test was applied. Test of normality (Kolmogorov-Smirnov) revealed non-normal distributions for the variables. Therefore, the Mann Whitney Test (U statistic) was used to compare the difference between the two groups. Multiple regression analysis was used to test if the SA factors (ACC of FC, CS, PS, VIS, SO. SR, ST, and ML, LP, and, SD as WF variables) significantly predicted participants' EFs (ACC of N-back, the number of cluster, perseveration errors in WCST, the accuracy of Go trials, and accuracy of No- Go trials in Go/No-Go task) in ADHD and TD groups.

\section{Results}

Basic information is shown in Table 2. There were no statistically significant differences for age $(z=-0.21, P=0.83)$, and educational grade $(z=$ $-0.48, P=0.63$ ) between the two groups. All Conner's Parent Rating Scale (ADHD symptoms) were significantly higher for ADHD group than TD group $(z=-9.64, P \leq 0.001)$. Furthermore, Chi-Square Test has shown that gender ratios between ADHD and TD groups do not differ $(P=0.081)$. 
Table 2

Basic information

\begin{tabular}{|llllll|}
\hline Variables & \multicolumn{2}{l}{ ADHD $(\mathrm{f}=\mathbf{1 4}, \mathrm{m}=\mathbf{5 0})$} & \multicolumn{2}{c|}{ TD $(\mathrm{f}=\mathbf{2 9}, \mathrm{m}=\mathbf{3 5})$} & \multirow{2}{*}{ z } \\
\cline { 2 - 5 } & Mean (SD) & Median & Mean (SD) & Median & \\
\hline Age & $10.02(0.98)$ & 10.02 & $9.97(0.99)$ & 10.01 & -0.21 \\
\hline Education grade & $4.09(1.16)$ & 4 & $3.95(0.99)$ & 4 & -0.48 \\
\hline Conner's scale & $67.73(4.30)$ & 67 & $26(14.23)$ & 24 & $-9.64^{*}$ \\
\hline *p <.001 & & & & \\
\hline Abbreviation. $\mathrm{f}=$ female, $\mathrm{m}=$ male, $\mathrm{SD}=$ standard deviation & \\
\hline
\end{tabular}

\section{Comparison of Spatial Ability variables between ADHD and TD groups}

The analyses showed that the children with ADHD were less accurate than the TD children in FC $(z=-2.09, P=0.036)$, CS $(z=-3.85, P=0.001)$, PS $(z=-5.17, P=0.001)$, VIS $(z=-5.22, P=0.001)$, SR $(z=-3.40, P=0.001)$, SO $(z=-3.75, P=0.001)$ tasks, and ML phase of WF task $(z=-3.39, P \leq$ $0.001)$. Number of trials in LP, and walked path in SD phase of WF task were more $(z=-2.69, P=0.007 ; z=-2.90, P=0.004)$ in $A D H D$ group (Table 3), besides ADHD and TD groups were significantly different in the RT variable of VIS $(z=-3.62, P=0.001), \mathrm{SR}(z=-5.20, P=0.001)$, and SO $(z=-2.84, P=0.005)$ tasks (Table 3$)$.

Table 3

Comparison of spatial ability variables between ADHD and TD groups.

\begin{tabular}{|c|c|c|c|c|c|c|c|}
\hline \multirow{2}{*}{\multicolumn{2}{|c|}{ Tasks }} & \multirow[t]{2}{*}{ Variables } & \multicolumn{2}{|l|}{ ADHD } & \multicolumn{2}{|l|}{ TD } & \multirow[t]{2}{*}{$\mathbf{z}$} \\
\hline & & & Mean (SD) & Median & Mean (SD) & Median & \\
\hline \multirow{2}{*}{\multicolumn{2}{|c|}{ Flexibility of closure task }} & $\operatorname{ACC}(\%)$ & $67.48(15.99)$ & 68.75 & $73.63(14.50)$ & 75.00 & $-2.09^{*}$ \\
\hline & & RT & 64.73 (12.99) & 66.09 & $63.06(18.40)$ & 60.53 & -1.70 \\
\hline \multirow{2}{*}{\multicolumn{2}{|c|}{ Closure speed task }} & $\operatorname{ACC}(\%)$ & $68.84(15.71)$ & 70.59 & $81.84(12.13)$ & 81.25 & $-3.85^{* *}$ \\
\hline & & RT & $84.81(28.05)$ & 78.9 & $75.57(17.42)$ & 75.98 & -1.58 \\
\hline \multirow{2}{*}{\multicolumn{2}{|c|}{ Perceptual speed task }} & $\operatorname{ACC}(\%)$ & $61.95(19.32)$ & 64.71 & $80.47(11.27)$ & 81.25 & $-5.17^{\star *}$ \\
\hline & & RT & $71.48(30.54)$ & 67.29 & $65.80(18.64)$ & 60.29 & -0.82 \\
\hline \multirow{2}{*}{\multicolumn{2}{|c|}{ Visualization Task }} & $\operatorname{ACC}(\%)$ & $44.44(17.43)$ & 44.44 & $61.81(15.41)$ & 61.67 & $-5.22^{* \star}$ \\
\hline & & RT & $59.94(27.08)$ & 63.84 & $80.53(28.65)$ & 79.04 & $-3.62^{* *}$ \\
\hline \multirow{2}{*}{\multicolumn{2}{|c|}{ Spatial relation Task }} & ACC (\%) & $61.52(17.21)$ & 58.33 & $72.56(20.56)$ & 75.00 & $-3.40^{* *}$ \\
\hline & & RT & $89.65(33.77)$ & 86.40 & $60.15(24.72)$ & 57.25 & $-5.20^{* *}$ \\
\hline \multirow{2}{*}{\multicolumn{2}{|c|}{ Spatial orientation Task }} & $\operatorname{ACC}(\%)$ & $43.75(20.27)$ & 38.89 & $56.51(19.61)$ & 61.11 & $-3.75^{* *}$ \\
\hline & & RT & 61.92(38.31) & 52.51 & $77.48(35.15)$ & 81.64 & $-2.84^{* *}$ \\
\hline \multicolumn{2}{|c|}{ Spatial temporal task } & $\operatorname{ACC}(\%)$ & $60.54(19.1)$ & 62.00 & $73.63(14.50)$ & 75.00 & -1.28 \\
\hline \multirow[t]{3}{*}{ Way finding Task } & $M L$ & ACC (\%) & 71.44 (9.19) & 73.00 & 79.59 (13.19) & 81.25 & $-3.39^{* *}$ \\
\hline & LP & NT (\%) & $52.50(12.97)$ & 50.00 & $46.87(7.75)$ & 50.00 & $-2.69^{* *}$ \\
\hline & $\mathrm{SD}$ & Meters & $36.20(7.02)$ & 35.37 & 32.21 (7.91) & 32.03 & $-2.90^{* *}$ \\
\hline \multicolumn{8}{|l|}{${ }^{*} p<.05, * * p<.001$} \\
\hline
\end{tabular}

\section{Comparison of Executive Functions variables between ADHD and TD groups}

According to Table 4 there were significant differences in all EFs variables between two groups, that is described as follows; ACC of N-back ( $z=$ -3.34, $P=0.001)$; number of Clusters $(z=-6.80, P=0.001)$, and perseveration error $(z=-6.59, P=0.001)$ of WCST; number of No-Go trials $(z=-5.46$, 
$P=0.001)$, number of Go trials $(z=-3.00, P=0.001)$, and Go speed $(z=-0.84, P=0.008)$ of Go/No-Go task between two groups.

Table 4

Comparison of executive function variables between ADHD and TD groups.

\begin{tabular}{|c|c|c|c|c|c|c|}
\hline \multirow[t]{2}{*}{ Tasks } & \multirow[t]{2}{*}{ Variables } & \multicolumn{2}{|l|}{ ADHD } & \multicolumn{2}{|l|}{ TD } & \multirow[t]{2}{*}{$\mathbf{z}$} \\
\hline & & Mean (SD) & Median & Mean (SD) & Median & \\
\hline N-Back & $\mathrm{ACC}(\%)$ & 79.27 (17.54) & 87.50 & 87.58 (13.92) & 95 & $-3.34^{\star \star}$ \\
\hline \multirow[t]{2}{*}{ WCST } & Perseveration error (\%) & $29.39(11.31)$ & 27.34 & $16.63(8.46)$ & 14.48 & $-6.59^{\star \star}$ \\
\hline & Clusters (\%) & $48.96(18.27)$ & 50 & $71.61(14.14)$ & 66.67 & $-6.80^{\star \star}$ \\
\hline \multirow[t]{3}{*}{ Go/No- Go } & No-Go (\%) & $81.14(17.63)$ & 80.77 & $94.73(7.21)$ & 96.15 & $-5.46^{\star \star}$ \\
\hline & Go (\%) & 84.83 (15.98) & 87.50 & $92.12(7.86)$ & 95.83 & $-3.00^{\star \star}$ \\
\hline & Go Speed (S) & $1.21(0.31)$ & 1.26 & $1.33(0.19)$ & 1.31 & $-0.84^{*}$ \\
\hline
\end{tabular}

\section{Correlation between Spatial Ability factors and Executive Functions in the two groups}

Table 5 illustrates the correlations between the variables in ADHD group. There were significant correlations between ACC of N-Back with ACC of FC $(r=0.33, P=0.008), \mathrm{CS}(r=0.55, P=0.001)$, PS $(r=0.27, P=0.034)$, VIS $(r=0.45, P=0.001)$, SO $(r=0.53, P=0.001)$, and ST $(r=0.31, P=0.013)$; number of Clusters of WCST with ACC of FC $(r=0.35, P=0.004)$, CS $(r=0.39, P=0.001)$, VIS $(r=0.28, P=0.027)$ and, SR $(r=0.37, P=0.003)$; perseveration error in WCST with CS $(r=-0.28, P=0.024)$; number of No-Go trials of Go/No-Go with ACC of CS $(r=0.30, P=0.015)$.

Table 5

Correlation between spatial ability factors and executive function in ADHD group.

\begin{tabular}{|c|c|c|c|c|c|c|c|c|c|c|c|}
\hline Tasks & Dependent Variables & FC & CS & PS & VIS & SR & so & ST & $M L$ & LP (NT) & SD (Meters) \\
\hline WCST & Perseveration Error & -0.21 & $-0.28^{*}$ & -0.08 & -0.24 & -0.09 & -0.11 & -0.04 & 0.15 & -0.12 & 0.04 \\
\hline \multirow[t]{2}{*}{ Go/No- Go } & No-Go & -0.04 & $0.30^{*}$ & 0.13 & 0.16 & 0.16 & 0.13 & 0.10 & -0.08 & -0.01 & 0.10 \\
\hline & Go & 0.04 & 0.12 & 0.10 & -0.07 & -0.12 & -0.01 & 0.10 & 0.12 & -0.14 & -0.05 \\
\hline
\end{tabular}

Also the correlations between the variables in TD group are described. There were significant correlations between ACC of N-Back with ACC of PS ( $r$ $=0.43, P=0.001)$, SR $(r=0.30, P=0.008), \mathrm{ML}(r=0.32, P=0.005)$, and LP $(r=-0.32, P=0.005)$; number of Clusters of WCST with ACC of VIS $(r=$ $0.41, P=0.001)$, ML $(r=0.35, P=0.002)$, and LP $(r=-0.46, P=0.001)$; perseveration error in WCST with VIS $(r=-0.36, P=0.002), \mathrm{ML}(r=-0.29, P=$ $0.011)$, and LP $(r=0.32, P=0.005)$; number of No-Go trials of Go/No-Go with ACC of FC $(r=-0.32, P=0.005)$, and number of Go trials with FC ( $r=$ $0.24, P=0.026)$, PS $(r=0.26, P=0.019)$, ST $(r=0.29, P=0.009)$, and ML $(r=0.36, P=0.002$, Table 6$)$.

Table 6

Correlation between spatial ability factors and executive function in TD group.

\begin{tabular}{|c|c|c|c|c|c|c|c|c|c|c|c|}
\hline Tasks & Dependent Variables & FC & CS & PS & VIS & SR & so & ST & ML & LP (NT) & SD (Meters) \\
\hline WCST & Perseveration Error & -0.22 & 0.01 & -0.09 & $-0.36^{\star \star}$ & -0.17 & -0.20 & 0.10 & $-0.29^{*}$ & $0.32^{\star \star}$ & -0.01 \\
\hline \multirow[t]{2}{*}{ Go/No- Go } & No-Go & $-0.32^{*}$ & -0.06 & -0.12 & 0.07 & 0.07 & -0.24 & -0.22 & -0.03 & -0.13 & 0.21 \\
\hline & Go & $0.24^{*}$ & 0.14 & $0.26^{*}$ & 0.16 & 0.05 & 0.14 & $0.29^{\star \star}$ & $0.36^{* *}$ & -0.10 & -0.02 \\
\hline
\end{tabular}


Multiple regression analyses were used to test if the SA factors significantly predicted the EFs in ADHD and TD groups.

\section{Working Memory}

A significant regression equation was found, $F=5.95, P<0.001$ with an $R^{2}$ adj $=0.440$. ACC of N-Back task was significantly predicted by ACC of these tasks in ADHD group: CS $(\beta=0.65, P=0.001)$, PS $(\beta=-0.33, P=0.039)$, SO $(\beta=0.33, P=0.019)$, and SD $(\beta=-0.31, p=0.008)$ of WF. Also, a significant regression equation was found for TD group, $F=2.89, P<0.001$ with an $R^{2}$ adj $=0.231$. ACC of N-Back task was significantly predicted by ACC of these tasks in TD group: PS $(\beta=0.35, P=0.015)$, and LP $(\beta=-0.24, P=0.048)$ of WF (Table 7).

Table 7

Comparison of predictability of spatial ability factors on working memory (N-Back task) in two groups.

\begin{tabular}{|c|c|c|c|c|c|c|c|c|c|c|c|c|c|}
\hline \multirow[t]{2}{*}{ Group } & \multicolumn{10}{|c|}{ Predictive Variables $(\mathbb{\nabla})$} & \multirow{2}{*}{$\mathrm{R}^{2}$} & \multirow{2}{*}{$\mathbf{R}^{2}{ }_{\text {adj }}$} & \multirow[t]{2}{*}{$\mathbf{F}$} \\
\hline & FC & CS & PS & VIS & SR & so & ST & $M L$ & LP & SD (Meters) & & & \\
\hline ADHD & 0.07 & $0.65^{\star *}$ & $-0.33^{*}$ & -0.08 & 0.06 & $0.33^{*}$ & 0.11 & 0.08 & 0.03 & $-0.31^{\star \star}$ & 0.529 & 0.440 & $5.95^{\star \star}$ \\
\hline TD & -0.12 & -0.07 & $0.35^{\star}$ & -0.07 & 0.26 & -0.10 & 0.14 & 0.14 & $-0.24^{*}$ & -0.09 & 0.353 & 0.231 & $2.891^{\star \star}$ \\
\hline
\end{tabular}

\section{Cognitive Flexibility}

A significant regression equation was found, $F=4.79, P<0.001$ with an $R^{2}$ adj $=0.375$. Number of clusters in WCST was significantly predicted by ACC of these tasks in ADHD group: FC $(\beta=0.28, P=0.033)$, CS $(\beta=0.68, P=0.001)$, SR $(\beta=0.35, P=0.008)$ and, SO $(\beta=-0.43, P=0.004)$. Also, a significant regression equation was found for TD group, $F=4.05, P<0.001$ with an $R^{2}$ adj $=0.326$. Number of clusters in WCST was significantly predicted by ACC of these tasks in TD group: VIS $(\beta=0.44, P=0.001)$, ML $(\beta=0.26, P=0.034)$, and LP $(\beta=-0.40, P=0.001)$ of WF (Table 8$)$.

Table 8

Comparison of predictability of spatial ability factors on cognitive flexibility (WCST) in two groups.

\begin{tabular}{|c|c|c|c|c|c|c|c|c|c|c|c|c|c|c|}
\hline \multirow{2}{*}{$\begin{array}{l}\text { Dependent } \\
\text { Variables }\end{array}$} & \multirow[t]{2}{*}{ Group } & \multicolumn{10}{|c|}{ 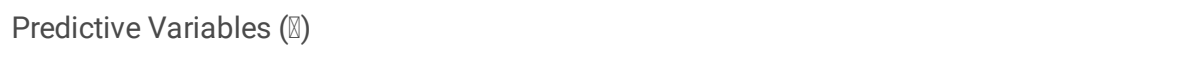 } & \multirow[t]{2}{*}{$\mathrm{R}^{2}$} & \multirow{2}{*}{$\mathrm{R}^{2}$ adj } & \multirow[t]{2}{*}{$\mathrm{F}$} \\
\hline & & FC & $\mathrm{CS}$ & PS & VIS & SR & so & ST & ML & LP & $\begin{array}{l}\text { SD } \\
\text { (Meters) }\end{array}$ & & & \\
\hline \multirow[t]{2}{*}{ Clusters } & ADHD & $0.28^{*}$ & $0.68^{\star *}$ & -0.13 & -0.10 & $0.35^{\star \star}$ & $-0.43^{\star *}$ & -0.09 & -0.16 & 0.03 & -0.23 & 0.475 & 0.375 & $4.79^{\star \star}$ \\
\hline & TD & 0.05 & -0.07 & -0.12 & $0.44^{\star *}$ & -0.09 & -0.08 & -0.03 & $0.26^{*}$ & $-0.40^{\star *}$ & -0.09 & 0.433 & 0.326 & $4.05^{\star *}$ \\
\hline \multirow{2}{*}{$\begin{array}{l}\text { Perseveration } \\
\text { Error }\end{array}$} & ADHD & -0.12 & $-0.53^{*}$ & 0.22 & 0.06 & -0.09 & 0.02 & 0.07 & 0.24 & -0.02 & 0.17 & 0.194 & 0.041 & 1.27 \\
\hline & TD & -0.22 & $0.31^{*}$ & 0.17 & $-0.31^{*}$ & -0.05 & -0.08 & 0.08 & -0.22 & $0.29^{*}$ & 0.01 & 0.335 & 0.209 & $2.67^{*}$ \\
\hline
\end{tabular}

The results indicated that the regression equation was not significant in the perseveration error variable in WCST task in ADHD group $(F=1.27, P=$ 0.270 with an $\left.R^{2}{ }_{a d j}=0.041\right)$. However, a significant regression equation was found for TD group, $F=2.67, P<0.05$ with an $R^{2}$ adj $=0.209$. The perseveration error was significantly predicted by ACC of these tasks in TD group: CS $(\beta=0.31, P=0.033), \mathrm{VIS}(\beta=-0.31, P=0.025)$, and $\mathrm{LP}(\beta=$ $0.29, P=0.020$ ) of WF (Table 8).

\section{Response Inhibition}

The results showed that the regression equation was not significant in the No-Go trials in $\mathrm{ADHD}$ group $\left(F=1.20, P=0.315\right.$ with an $\left.R^{2}{ }_{a d j}=0.030\right)$ and also, in TD group $\left(F=1.79, P=0.84\right.$ with an $R^{2}$ adj $=0.112$, Table 9$)$. Furthermore, the results indicated that the regression equation was not noteworthy in the Go trials in $\operatorname{ADHD}$ group $\left(F=0.96, P=0.484\right.$ with an $\left.R^{2}{ }_{\text {adj }}=-0.006\right)$ and also, in TD group $\left(F=1.51, P=0.162\right.$ with an $R^{2}{ }_{\text {adj }}=0.075$, Table 9). 
Table 9

Comparison of predictability of spatial ability factors on inhibition (Go/No- Go Task) in two groups.

\begin{tabular}{|c|c|c|c|c|c|c|c|c|c|c|c|c|c|c|}
\hline \multirow[t]{2}{*}{ Dependent Variables } & \multirow[t]{2}{*}{ Group } & \multicolumn{10}{|c|}{ 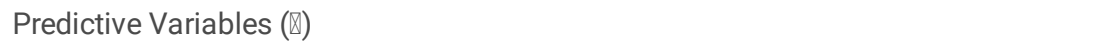 } & \multirow[t]{2}{*}{$R^{2}$} & \multirow[t]{2}{*}{$\mathrm{R}^{2}{ }_{\mathrm{adj}}$} & \multirow[t]{2}{*}{$\mathrm{F}$} \\
\hline & & FC & cs & PS & VIS & SR & so & ST & ML & LP & SD & & & \\
\hline \multirow[t]{2}{*}{ No/Go } & ADHD & -0.23 & $0.53^{*}$ & -0.15 & -0.05 & 0.16 & 0.04 & 0.01 & -0.21 & -0.04 & -0.06 & 0.184 & 0.030 & 1.20 \\
\hline & TD & $-0.36^{*}$ & 0.05 & 0.07 & 0.16 & 0.16 & -0.22 & -0.09 & 0.01 & -0.13 & 0.17 & 0.253 & 0.112 & 1.795 \\
\hline \multirow[t]{2}{*}{ Go } & ADHD & 0.11 & 0.37 & -0.02 & -0.22 & -0.19 & -0.20 & 0.14 & 0.17 & -0.18 & -0.11 & 0.154 & -0.006 & 0.965 \\
\hline & TD & 0.16 & -0.04 & 0.05 & 0.11 & -0.03 & -0.13 & 0.24 & 0.28 & -0.04 & -0.02 & 0.22 & 0.07 & 1.509 \\
\hline
\end{tabular}

\section{Discussion}

The present study was designed with two major goals. Firstly, we attempted to clarify the difficulties in eight factors of SA in children with ADHD. Secondly, we evaluated the predictability of SA on the EFs. The results highlighted that the performance of children with ADHD was poorer than TD in all the eight factors of SA except ST. Furthermore, the mean of RT in VIS, SR, and SO tasks was more in children with ADHD. We also found out some of the SA factors not only were correlated with EFs but also predicted them in ADHD and TD children.

\section{Spatial Ability In Children With ADHD}

We measured all SA factors in the present study. We explored that ADHD children had more difficulty in performing all of the SA tasks, comparing to the TD group. On the other hand, children with ADHD had deficits in static-intrinsic, dynamic-intrinsic, static-extrinsic and dynamic-extrinsic skills based on Uttal classification (9).

In line with our study, it was reported that children with ADHD had problems in the FC factor which was measured with an Embedded Figure test $(30,37)$ and SR factor which was examined with the Mental Rotation Moomies test in Jakobson and Kikas (29) study, and the Turning test and the Spatial Relation test in Aman and colleagues study (26). All of these tasks together with the SR task were employed in the current study to examine the ability of mental rotation of figures. In contrast with our study, when the Gestalt Closure task was applied for measuring CS factor, in Kalff's (30), and Mariani and Barkley's studies (39), children with ADHD did not perform differently from TD children. One possible reason for this contradiction can be related to the level of difficulty of the Gestalt which is more difficult than the Embedded Figure test and our CS task for children. In the literature review, we found that some studies investigated spatial ability with different terms and measured this ability with inappropriate tests such as the Benetton judgment line orientation, Benetton visual retention, and Rey Osterrieth Complex Figure that were not specific for each factor of spatial ability. It's worthy to note that none of the above-mentioned tests are related to the nature of spatial ability factors $(25,28,31,32,34,35$, and $40-42)$. At the neural level, anatomical and functional neuroimaging studies have revealed the difference in the parietal cortex especially the IPL area in children with ADHD comparing to normal children $(18,19,61$, and 62$)$. The parietal cortex particularly the IPL, has known to be involved in many aspects of spatial processing and manipulation $(14,15)$. These findings may associate spatial ability problems in ADHD group with an abnormality in the IPL.

Regarding the ST task, significant differences were not found between the two groups. In tasks that measure ST ability such as our task, participants are asked to predict not only where several moving objects will interrupt, but also to decide when this interception might occur. Thus, this task is to evaluate the ability to represent time and use this information to calculate relative velocity, which is then used to extrapolate a viable intercept, and to make an appropriate spatial judgment (63). Consequently, it can be inferred that performing the ST task needs the participant to divide his/her attention since the ability to attend and respond to multiple tasks or task demands simultaneously divided attention (64). The fact that children with ADHD have been reported to perform similar to, or better than children without ADHD on tasks that require divided attention, can be the reason for a similar performance on the ST tasks in ADHD and TD groups (65).

One of our findings was related to differences between the mean of RT in the SA tasks in two groups of participants. In regards to the results, children with ADHD consumed more reaction time than TD children in the VIS, SR, and SO tasks. SA factors are highly consistent with both the hierarchical models e.g., Carroll (66), and the nonhierarchical Radex models of human intelligence(67). Both models suggest a complexity continuum along which cognitive tasks can be organized. In these models, the more complex a task is, the more strongly it tends to be correlated with general intelligence $(G)$ and the higher it is placed in the hierarchy (in the hierarchical models) or the closer it is placed to the center of the configuration (in the Radex models). Besides, this complexity continuum nicely corresponds to the degree of central executive involvement. From this perspective, more complex tasks such as the VIS, SR, and SO compared with the FC, CS, and PS tasks need more time for solving their items. Since ADHD children, in general, have slower processing performance comparing to the typical population $(68,69)$, it seems logical that they need more time to response more difficult tasks. 


\section{Prediction Of Executive Functions With Spatial Ability Factors}

Considering our findings, children with ADHD performed all EFs tasks poorer than TD children. These results frequently proved in previous studies $(70,71)$. Based on the second our aim, results indicated that working memory and cognitive flexibility were predicted by some SA factors in ADHD children.

The regression analysis revealed that the working memory can be predicted by ACC of CS, PS, SO, and SD phase of WF tasks. These results are well justified by Baddeley and Hitch's working memory theory (72). The theory proposed a model containing three components: the central executive, the phonological loop, and the visuospatial sketchpad. The CS factor is concerned with the speed of apprehending and identifying a visual pattern, often in the absence of complete stimuli. Performance on the CS tasks (such our task) is likely to be aided by strategic mental searches of possible shapes, and hence the contribution of visuospatial and executive components of working memory theory may be relatively high. The PS factor was defined as the speed in finding figures and making comparisons between them. The concept of PS can be considered to be the centroid of several sub-factors (73). The sub-factors have been defined as the speed of symbol discrimination, speed of making comparisons and speed of form discrimination as in recognizing predetermined, but novel configurations (74). The ability to maintain spatial representations may be essential ingredients of all these sub factors. SO factor requires considerable reasoning skill and participants may solve items by mentally rotating and manipulating them rather than moving an image of the self to the desired perspective. Therefore, it requires maintaining orientation a visual pattern and manipulating its mental representation in the mind. It can be deduced that two of the three components of working memory theory (visuospatial sketchpad stores and central executive) involve in solving SO tasks. Working memory includes holding information in mind and the SD phase of the WF task needs to hold possible paths information and to choose the shortest. Taken together, the prediction of working memory by the CS, PS, SO, and SD in our study was not unexpected.

Our findings indicated that cognitive flexibility is predicted by ACC of the SO, FC, CS, and SR tasks. The ability to change perspectives spatially is known to be one of the aspects of cognitive flexibility, concerning this (e.g., "What would this look like if I viewed it from a different direction?" fact (3), the prediction of cognitive flexibility by SO factor in ADHD children is logical in the current study. We also found out that children's performance in the FC and CS tasks predicted the performance of them in WSCT. For an explanation of results, it may point to visual global and local processing. To solve FC problems, children should find a hidden specific stimulus in a complex and ambiguous visual pattern. Finding a specific stimulus depends on the ability to shift global processing to local processing, however children in CS tasks have to switch from local to global processing to recognize incomplete stimulus. Since each item of FC and CS tasks contain four complex patterns, children must shift processing quickly among the patterns. Furthermore, the answer to each trial of two tasks is consistent with the nature of cognitive flexibility. Finally, SR factor was able to predict cognitive flexibility. This factor is defined by the tasks in which participants are required to determine whether the two presented stimuli (animals in the current task) are identical or mirror images of each other. To overcome the challenge, the participant should frequently switch between upright and rotated positions of animal in mind to understand two animals are the same or different, so cognitive flexibility partly involves in solving the task. According to what was explained, it is logical to predict cognitive flexibility by mentioned spatial factors.

In our study, although children with ADHD had a deficit in inhibitory control, this cognitive function was not predicted by any of the SA factors. Inhibition is defined as the ability to revoke or suppress an action which is irrelevant, no longer needed, and/or inappropriate (75-77). Inhibition is known to be divided into 2 subgroups of pre-potent response and resistance to distractor interference. However, the third component of inhibition is defined by some as the coined resistance to proactive interference $(76,78)$. In the current study, we focused on pre-potent response inhibition and utilized the Go/No-Go task for measuring it. Our spatial ability tasks didn't require to suppress a pre-potent stimulus, but it is not unlikely that interference and proactive inhibition at least relate to some of the SA factors.

This is the first regression analysis that examines the prediction of EFs by SA factors on in ADHD children. Therefore, there is a requirement for our findings to be confirmed through the next researches. The results of our study indicated that children with ADHD are less accurate than TD children in the three EFs tasks and also different aspects of the SA were related to major EFs tasks. Furthermore, they could predict the performance of children with ADHD in two EFs tasks. These behavioral findings are in correspondence with neuroimaging evidence about inter-correlation between the parietal and frontal coteries $(19,62$, and 79$)$.

The other finding of this part of the study indicated that in TD as well as children with ADHD some of the spatial ability factors were able to predict their performance in both working memory and cognitive flexibility. That way, the PS was able to predict working memory and ACC of the CS, VIS, $\mathrm{ML}$, and LP of WF tasks were able to predict cognitive flexibility. Spatial ability factors in children with ADHD were not able to predict response inhibition, this result was the same in TD children. Since the existence of functional relationships and interactions between the parietal cortex and the prefrontal cortex has been confirmed, predicting the performance of TD children in some spatial factors on working memory, and cognitive flexibility is rational. But the reason why different spatial factors in the two groups were able to predict the performance of these two executive functions is probably due to the unusual interactions between the impaired prefrontal cortex and the parietal cortex in children with ADHD (19, 62 , and 79) and also can be to attributable to anatomical and clinical heterogeneity in this disorder (80). Functional neuroimaging studies are required in both ADHD and TD children separately in order to an accurate understanding of this subject. 


\section{Limitations And Future Directions}

Concerning the current study, the following limitations are noteworthy. Firstly, ADHD is a clinically heterogeneous disorder with a high rate of comorbid conditions, thus making it extremely difficult to completely control the comorbidity of a representative sample of children with ADHD. In the present study, ADHD was the main diagnosis, without any other diagnosis, although comorbid emotional or behavioral symptomatology may exist in some cases, without constituting a disorder in themselves. Secondly, the sample size was small, specifically for regression analysis and also the ADHD group was limited by the low number of females among the participants. Thus, the results of this study may be more applicable to the male population with ADHD, which is the population that most frequently suffer this disorder.

Since our study showed that at least some of the SA factors and EFs impairments were related to each other and SA impairment reflected the disruption of EFs, we suggest to examine and report the effectiveness of various spatial training programs not only on the enhancement of spatial factors but also EFS in people with ADHD. Besides, functional neuroimaging studies on ADHD people should take these cognitive functions into account.

\section{Conclusion}

Our study adds to this research field results in favor of the existence of alterations in the spatial ability of children with ADHD. To our knowledge, this was the first study that investigated SA deficits comprehensively in children with ADHD. On the other hand, we proved that some SA factors

affect EFs. We can conclude that our study supported the hypothesis that EFs deficits are an important component of the ADHD neuropsychology, although they are not sufficient to fully explain its symptomatology (81). From a clinical point of view and regarding the importance of SA in daily activities, for instance, academic skills during childhood, it seems to be necessary for experts to include assessment of all SA factors and training them in cognitive rehabilitation programs for children suffering from ADHD.

\section{Declarations}

\section{Ethics approval and consent to participate}

The study was approved by the ethical committee of the Shahid Beheshti University (ethical code: IR.SBU.ICBS.97/1022).

\section{Consent for publication}

The authors consent for this paper to be published.

\section{Availability of data and materials}

All data and material are available at the Institute for Cognitive and Brain Sciences at the Shahid Beheshti University. These data will be used for another research in the near future.

\section{Competing interests}

The authors declare that the research was conducted in the absence of any commercial or financial relationships that could be construed as a potential conflict of interest.

\section{Funding}

Not applicable.

\section{Authors' contributions}

SS and VN contributed in design, SS gathered data, SS and JF analyzed data. SS and VN contributed to drafting the manuscript. All authors read and approved the final manuscript.

\section{Acknowledgements}

Not applicable.

\section{References}


1. Association American Psychiatric. Diagnostic and statistical manual of mental disorders. BMC Med. 2013;17:133-7.

2. Faraone SV. Genetics of attention de fi cit hyperactivity disorder. Mol Psychiatry. 2019;24:562-75.

3. Diamond A. Exectuve functions. Annu Rev Psychol [Internet]. 2013;64:135-68. Available from: https://www.ncbi.nlm.nih.gov/pmc/articles/PMC4084861/pdf/nihms-602706.

4. Kurtz LA. Visual perception problems in children with $A D / H D$, autism, and other learning disabilities: A guide for parents and professionals. Jessica Kingsley Publishers; 2006 Apr 13.

5. 10.1016/B978-0-12-394388-0.00006-X

Mix KS, Cheng YL. The Relation Between Space and Math. Developmental and Educational Implications [Internet]. Vol. 42, Advances in Child Development and Behavior. Elsevier Inc.; 2012. 197-243 p. Available from: http://dx.doi.org/10.1016/B978-0-12-394388-0.00006-X.

6. Newcombe NS, Shipley TF. Thinking about spatial thinking: New typology, new assessments. InStudying visual and spatial reasoning for design creativity 2015 (pp. 179-192). Springer, Dordrecht..

7. Verdine BN, Golinkoff RM, Hirsh-Pasek K, Newcombe NS. I. Spatial skills, their development, and their links to mathematics. Monographs of the society for research in child development. 2017 Mar;82(1):7-30.

8. Linn MC, Petersen AC. Emergence and characterization of sex differences in spatial ability: a meta-analysis. Child Dev [Internet]. 1985;56(6):1479-98. Available from: http://www.ncbi.nlm.nih.gov/pubmed/4075870.

9. Uttal DH, Meadow NG, Tipton E, Hand LL, Alden AR, Warren C, et al. The malleability of spatial skills: A meta-analysis of training studies. Psychol Bull. 2013;139(2):352-402.

10. Yilmaz HB. On the development and measurement of spatial ability. International Electronic Journal of Elementary Education. 2009;1(2):8396.

11. Raz A. Anatomy of attentional networks. Anat Rec - Part B New Anat. 2004;281(1):21-36.

12. Berger A, Posner MI. Pathologies of brain attentional networks. Neurosci Biobehav Rev. 2000;24:3-5.

13. Heil M, Jansen-Osmann P. Children's left parietal brain activation during mental rotation is reliable as well as specific. Cogn Dev. 2007;22(2):280-8.

14. Sack AT. Parietal cortex and spatial cognition. Behav Brain Res. 2009;202(2):153-61.

15. Gazzaniga MS. The cognitive neurosciences. MIT press; 2009.

16. Nakao T, Radua J, Rubia K, Mataix-Cols D. Gray matter volume abnormalities in ADHD: Voxel-based meta-analysis exploring the effects of age and stimulant medication. Am J Psychiatry. 2011;168(11):1154-63.

17. Filipek PA, Steingard RJ, Renshaw PF. Volumetric MRI analysis comparing subjects having attention-deficit hyperactivity disorder with normal controls. Neurology. 1997;48:589-601.

18. Vance A, Silk TJ, Casey M, Rinehart NJ, Bradshaw JL, Bellgrove MA, et al. Right parietal dysfunction in children with attention deficit hyperactivity disorder, combined type : A functional MRI study Right parietal dysfunction in children with attention deficit hyperactivity disorder, combined type : a functional MRI study. Mol Autism. 2007;(October):1-7.

19. Silk S, Vance TJ, Rinehart A, Bradshaw N, Cunnington JL. R. Dysfunction in the fronto-parietal network in attention deficit hyperactivity disorder (ADHD): an fMRI study. Brain Imaging and Behavior. 2008 Jun 1;2(2):123-31.

20. Fan LY, Gau SSF, Chou TL. Neural correlates of inhibitory control and visual processing in youths with attention deficit hyperactivity disorder: A counting Stroop functional MRI study. Psychol Med. 2014;44(12):2661-71.

21. Barkley RA. Behavioral Inhibition. Sustained Attention, and Executive Functions : Constructing a Unifying Theory of ADHD. Psychol Bull. 1997;121(1):65-94.

22. Castellanos FX, Tannock R. Neuroscience of attention-deficit/hyperactivity disorder: The search for endophenotypes. Nat Rev Neurosci. 2002;3(8):617-28.

23. Pennington BF, Ozonoff S. Executive Functions and Developmental Psychopathology. j Child Psychol Psychiat. 1996;37(I):51-87.

24. Schachar R, Mota VL, Logan GD, Tannock R, Klim P. Confirmation of an inhibitory control deficit in attention-deficit/hyperactivity disorder. J Abnorm Child Psychol. 2000;28(3):227-35.

25. 10.1016/j.sbspro.2015.01.878

Alpanda S. The investigation of the relationship between ADHD and visual- spatial functions. Procedia - Soc Behav Sci [Internet]. 2015;174:2219-25. Available from: http://dx.doi.org/10.1016/j.sbspro.2015.01.878.

26. Aman CJ, Roberts RJ, Pennington BF. A Neuropsychological Examination of the Underlying Deficit in Attention Deficit Hyperactivity Disorder: Frontal Lobe Versus Right Parietal Lobe Theories. Dev Psychol. 1998;34(5):956-69.

27. Chelune GJ, Ferguson W, Koon R, Dickey TO. Frontal lobe disinhibition in attention deficit disorder. Child Psychiatry Hum Dev. 1986;16(4):22134. 
28. Garcia-Sanchez C, Armando E, Suarez-Romero E, Junque C. Right Hemisphere Dysfunction in Subjects With Attention-Deficit Disorder With and Without Hyperactivity. j Child Neural. 1997;12:107-15.

29. Jakobson A, Kikas E. Cognitive Functioning in Children With and Without Attention Deficit/Hyperactivity Disorder With and Without Comorbid Learning Disabilities. J Learn Disabil. 2007;40(3):194-202.

30. Kalff AC, Hendriksen JGM, Kroes M, Vles JSH, Steyaert J, Feron FJM, et al. Neurocognitive Performance of 5- and 6YearOld Children Who Met Criteria for Attention Deficit / Hyperactivity Disorder at 18 Months Follow-Up: Results from a Prospective Population S ... Neurocognitive Performance of 5- and 6-Year-Old Children Who Met Cr. J Abnorm Child Psychol. 2002;30(6):589-98.

31. Kibby MY, Cohen MJ, Hynd GW. Clock face drawing in children with attention-deficit/hyperactivity disorder. Archives of clinical neuropsychology. 2002 Aug 1;17(6):531 - 46.

32. Risser MG, Bowers TG. Cognitive and neuropsychological characteristics of Attention Ddeficit Hyperactivity Disorder children receiving stimulant medications. Percept Mot Skills. 1993;77:1023-31.

33. $10.1016 /$ j.ridd. 2012.02 .010

Shen I, Lee T, Chen C. Research in Developmental Disabilities Handwriting performance and underlying factors in children with Attention Deficit Hyperactivity Disorder. Res Dev Disabil [Internet]. 2012;33(4):1301-9. Available from: http://dx.doi.org/10.1016/j.ridd.2012.02.010.

34. 10.1097/00004583-199007000-00006 Loge DV, Staton RD, Ph D, Beatty WW, Ph D. Performance of Children with ADHD on Tests Sensitive to Frontal Lobe Dysfunction. J Am Acad Child Adolesc Psychiatry [Internet]. 1986;29(4):540-5. Available from: http://dx.doi.org/10.1097/00004583-199007000-00006.

35. Leitner Y, Doniger GM, Barak R, Simon ES, Hausdorff JM. Cognitive Assessment for Attention-Deficit Hyperactivity Disorder: Evidence for Deficits. J Child Neurol. 2007;22(3):264-76.

36. Geurts HM, Vert S, Roeyers H, Sergeant JA. ADHD subtypes: do they differ in their executive functioning profile ? Arch Clin Neuropsychol. 2005;20:457-77.

37. Cohen NJ. Cognitive Styles in Adolescents Previously Diagnosed as Hypefactive *.j Child Psychol Psychiat. 1972;13(April):203-10.

38. Ahmetoglu E, Ahyan NA. A. A Comparative Study on the Visual Perception of Children with Attention Deficit Hyperactivity Disorder. J Appl Sci. 2008;8(5):830-5.

39. Mariani MA, Barkley RA. Developmental Neuropsychology Neuropsychological and academic functioning in preschool boys with attention deficit hyperactivity disorder. Dev Neuropsychol. 1997;13(1):11-29.

40. Semrud-clikeman M, Walkowiak J, Christopher G. Neuropsychological Differences Among Children With Asperger Syndrome, Nonverbal Learning Disabilities, Attention Deficit Disorder, and Controls. Dev Neuropsychol. 2010;35(5):582-600.

41. Pitcher TM. Motor performance and motor control in children with subtypes of attention deficit hyperactivity disorder. Curtin University of Technology.; 2001.

42. Kibby MY, Dyer SM, Vadnais SA, Jagger AC, Casher GA, Stacy M. Visual processing in reading disorders and attention-deficit / hyperactivity disorder and its contribution to basic reading ability. Front Psychiatry. 2015;6(October):1-11.

43. Conners k. c., Sitarenios G, Parker JDA, Epstein JN. The revised Conners' Parent Rating Scale (CPRS-R): Factor structure, reliability, and criterion validity. J Abnorm Child Psychol. 1998;26(4):257-68.

44. Khoushabi K. Prevalence of attention deficit hyperactivity disorder and its comorbidity disorders in elementary students in Tehran. University of Social Welfare and Rehabilitation Sciences; 2002.

45. Soluki S, Yazdanl S, Arjmandnia AA, Fathabadi J, Hassanzadeh S, Nejati V, et al. Comprehensive assessment of spatial ability in children: a computerized tasks battery. Advances in Cognitive Psychology. 2020;[In Press].

46. Gardner MF. The Test of Visual-Perceptual Skills (Non-Motor): Revised Manual. Hydesville: Psychological and Educational Publications.Inc; 1996.

47. Harris J, Newcombe NS, Hirsh-pasek K. A New Twist on Studying the Development of Dynamic Spatial Transformations: Mental Paper Folding in Young Children. MIND. BRAIN Educ. 2013;7(1):49-55.

48. Wiedenbauer G, Jansen-Osmann P. Manual training of mental rotation in children. Learning and instruction. 2008 Feb 1;18(1):30-41.

49. Zoest V Van. Relationship between video gaming and spatial thinking skills of children 8 to 10 years old Vera. Wageningen University and Research Centre.2015.

50. Hegarty M, Waller D. A dissociation between mental rotation and perspective-taking spatial abilities. Intelligence. 2004;32:175-91.

51. 10.1016/j.learninstruc.2013.12.007

Sanchez CA, Wiley J. The role of dynamic spatial ability in geoscience text comprehension. Learning and Instruction. 2014 Jun 1;31:33-45..

Available from: http://dx.doi.org/10.1016/j.learninstruc.2013.12.007.

52. Mengue-Topio H, Courbois Y, Farran EK, Sockeel P. Route learning and shortcut performance in adults with intellectual disability: A study with virtual environments. Res Dev Disabil. 2011;23(1):345-52. 
53. Jonides J, Schmacher E, Smith E, Lauber E, Awh E, Minoshima S, et al. Verbal Working Memory Load Affects Regional Brain Activation as Measured PET. J Cogn Neurosci. 1997;9(4):462-75.

54. Kane MJ, Engle R. The role of prefrontal cortex in working-memory capacity, executive attention, and general fluid intelligence : An individualdifferences perspective. Psychon Bull Rev. 2002;9(4):637-71.

55. Jaeggi SM, Buschkuehl M, Perrig WJ, Meier B. The concurrent validity of the N-back task as a working memory measure. Memory. 2010;18(4):394-412.

56. Grant DA, Berg E. A behavioral analysis of degree of reinforcement and ease of shifting to new responses in a Weigl-type card-sorting problem. J Exp Psychol. 1948;38(4):404.

57. Axelrod BN, Woodard JL, Henry RR. Analysis of an abbreviated form of the Wisconsin Card Sorting Test. Clin Neuropsychol. 1992;6(1):27-31.

58. Bowden SC, Fowler KS, Bell RC, Whelan G, Clifford CC, Ritter AJ, et al. The Reliability and Internal Validity of the Wisconsin Card Sorting Test. Neuropsychol Rehabil. 1998;8(3):243-54.

59. Douglas VI, Baker AG. Effects of Reward and Response Costs on Inhibition in ADHD Children. J Abnorm Psychol. 1995;104(1):232-40.

60. Newman JP. Passive Avoidance in Syndromes of Disinhibition. Passive Avoidance in Syndromes of Disinhibition : Psychopathy and Extraversion. J Pers. 1985;48(5):1316-27.

61. Durston S, Davidson MC, Mulder MJ, Spicer JA, Galvan A, Tottenham N, et al. Neural and behavioral correlates of expectancy violations in attention-deficit hyperactivity disorder. J Child Psychol Psychiatry Allied Discip. 2007;48(9):881-9.

62. Silk T, Vance A, Rinehart N, Egan G, O’Boyle M, Bradshaw JL, et al. Fronto-parietal activation in attention-deficit hyperactivity disorder, combined type: Functional magnetic resonance imaging study. Br J Psychiatry. 2005;187(SEPT.):282-3.

63. Hunt E, Pellegrino JW, Frick RW, Farr SA, Alderton D. The ability to reason about movement in the visual field. Intelligence. 1988;12(1):77-100.

64. Barkley RA. Attention-deficit hyperactivity disorder: A handbook for diagnosis and treatment. 4th ed. New York: Guilford Publications; 2014.

65. Elosúa R, Olmo S, Del, Contreras MJ, Miranda A. Differences in Executive Functioning in Children with Attention Deficit and Hyperactivity Disorder (ADHD). Front Psychol. 2017;8:1-11.

66. Carroll JB. Human cognitive abilities: A survey of factor-analytic studies. Cambridge University Press; 1993.

67. Marshalek B, Lohman DF, Snow RE. The Complexity Continuum in the Radex and. Hierarchical Models of Intelligence *. Intelligence. 1983;7:107-27.

68. Alderson RM, Rapport MD. ADHD and Behavioral Inhibition: A Re-examination of the Stop-signal Task. J Abnorm Child Psychol. 2008;36(June):989-98.

69. Kofler MJ, Rapport MD, Sarver DE, Raiker JS, Orban SA, Friedman LM, et al. Reaction time variability in ADHD: a meta- analytic review of 319 studies. University of Central Florida; 2013.

70. Gau SS, Shang C. Executive functions as endophenotypes in ADHD: evidence from the Cambridge Neuropsychological Test Battery (CANTAB). J Child Psychol Psychiatry. 2010;51(7):838-49.

71. McAuley T, Crosbie J, Charach A, Schachar R. The persistence of cognitive deficits in remitted and unremitted ADHD: A case for the stateindependence of response inhibition. J Child Psychol Psychiatry. 2014;55(3):292-300.

72. Baddeley AD, Hitch GJ. Developments in the Concept of Working Memory. Neuropsychology. 1994;8(4):485-93.

73. Bechtoldt HP. Factor analysis of the Airman Classification Battery with civilian reference tests. Human Resources Research Center, Air Research and Development Command; 1953.

74. French JW, Ekstrom RB, Price LA. Manual for kit of reference tests for cognitive factors (revised 1963). Educational Testing Service Princeton NJ; 1963.

75. 10.1016/j.biopsych.2010.07.024

Aron AR. From reactive to proactive and selective control: developing a richer model for stopping inappropriate responses. Biological psychiatry. 2011 Jun 15;69(12):e55-68.. Available from: http://dx.doi.org/10.1016/j.biopsych.2010.07.024.

76. Friedman NP, Miyake A. The Relations Among Inhibition and Interference Control Functions: A Latent-Variable Analysis. J Exp Psychol. 2004;133(1):101-35.

77. Nigg JT. On Inhibition / Disinhibition in Developmental Psychopathology: Views From Cognitive and Personality Psychology and a Working Inhibition Taxonomy. Psychol Bull. 2000;126(2):220-46.

78. Miyake A, Friedman NP, Emerson MJ, Witzki AH, Howerter A, Wager TD. The Unity and Diversity of Executive Functions and Their Contributions to Complex "' Frontal Lobe"' Tasks: A Latent Variable Analysis and. Cogn Psychol. 2000;41:49-100.

79. Arnsten AF. Toward a new understanding of attention-deficit hyperactivity disorder pathophysiology. CNS drugs. 2009 Nov 1;23(1):33-41.

80. Samea F, Soluki S, Nejati V, Zarei M, Cortese S, Eickhoff SB, et al. Brain alterations in children/adolescents with ADHD revisited: A neuroimaging meta-analysis of 96 structural and functional studies. Neurosci Biobehav Rev. 2019;100:1-8. 
81. Willcutt EG, Doyle AE, Nigg JT, Faraone SV, Pennington BF. Validity of the executive function theory of attention-deficit/ hyperactivity disorder: A meta-analytic review. Biol Psychiatry. 2005;57(11):1336-46.

\section{Supplementary Files}

This is a list of supplementary files associated with this preprint. Click to download.

- Tables.docx 